\title{
Ärzteschaft und Industrie
}

Im angelsächsischen Raum werden Ärzteschaft und Industrie als «uneasy bedfellows» bezeichnet. Der FMH-Zentralvorstand hat 1990 erste Empfehlungen zu dieser Problematik in der Ärztezeitung veröffentlicht. Die FMH-Standesordnung von 1997 hält einige wichtige Grundsätze fest.

Eine Arbeitsgruppe der Qualitätssicherungskommission der Schweizerischen Gesellschaft für Gynäkologie und Geburtshilfe hat sich in Absprache mit dem FMH-Zentralvorstand in verdankenswerter Weise mit der Thematik beschäftigt.

Die ersten Empfehlungen, die nachfolgend veröffentlicht werden, beschränken sich auf das vordringlichste Thema: Das revidierte Schweizer Strafrecht verlangt eine rasche und kritische Überprüfung und Offenlegung der Beziehungen zwischen Industrie und dem Service Public.

Ein grosser Dank gebührt Herrn Prof. Urs Haller, Präsident der Qualitätskommission der Schweizerischen Gesellschaft für Gynäkologie und Geburtshilfe, sowie Dr. Gero Drack, Präsident der Subkommission "Ärzteschaft und Industrie», für den sehr engagierten Einsatz bei der Ausarbeitung der dringend benötigten und nun vorliegenden Orientierungshilfe.

Dr. H. H. Brunner, Präsident FMH

\section{Drittmittel}

\section{und Sponsoring}

\section{Zur Beziehung zwischen der Ärzteschaft und der Industrie}

Subkommission "Ärzteschaft und Industrie" der Kommission Qualitätssicherung SGGG*

\section{Das Problem}

Die Zusammenarbeit zwischen der Ärzteschaft und der Industrie stellte und stellt ein wesentliches, früher kaum bestrittenes Element in der medizinischen Forschung, in der klinischen Medizin und im Gesund-

\footnotetext{
* Für die Subkommission Ärzteschaft und Industrie: Dr. med. MPH Gero Drack, St. Gallen Für die Kommission Qualitätssicherung SGGG: Prof. Dr. med. Urs Haller, Zürich Mitglieder der Subkommission:

Prof. Dr. med. Alfonso Almendral, Basel; Dr. med. Peter Fehr, Schaffhausen; Dr. med. Peter Diego Hagmann, Zürich; Prof. Dr. med. Patrick Hohlfeld, Lausanne; Prof. Dr. med. Mario Litschgi, Schaffhausen; Dr. med. Judith Pok, Zürich; Dr. med. Jacques Seydoux, Delémont; Dr. med. Michael Singer, Zürich. Schweizerische Patientenorganisation: Margrit Kessler, Altstätten, Präsidentin SPO. Industrie: Dr. Martin Batzer / Marcel Marolf, Novartis Pharma Schweiz AG, Bern. Juristischer Beirat: Fürsprecher Hanspeter Kuhn, Bern.
}

Korrespondenz:

Prof. Dr. med. Urs Haller

Universitätsspital

Departement für Frauenheilkunde

Frauenklinikstrasse 10

CH-8091 Zürich heitswesen dar. Bei der Entwicklung und Einführung neuer Diagnostika und Therapeutika sind Kliniken und Firmen aufeinander angewiesen. Aus ökonomischer Sicht bildet in der Regel die Industrie den stärkeren Partner. Manche bisher durchaus akzeptierten Formen von Zuwendungen von Firmen bzw. Dritten an Kliniken und Ärzte werden in den letzten Jahren in verschiedenen Ländern zunehmend kritisch hinterfragt. Es liegen ausländische Gerichtsurteile vor, welche in der Ärzteschaft zu Überraschung, Verunsicherung und teilweise heftigen Reaktionen geführt haben.

Die Kommission Qualitätssicherung der SGGG hat sich des Themenkomplexes angenommen. Im vorliegenden Expertenbrief will sie mit Nachdruck auf die ethischen und rechtlichen Dimensionen des Themas hinweisen.

- Besonders dringlich erscheint der Handlungsbedarf an den öffentlichen Spitälern. Hier sind die Beziehungen zur Industrie auf ihre Vereinbarkeit mit den neuen Bestimmungen des Korruptionsstrafrechts ${ }^{1}$ zu überprüfen. Der Expertenbrief formuliert dazu konkrete Empfehlungen.

- Eine ausführlichere Stellungnahme der Kommission wird folgen. Sie wird sich mit der Frage auseinandersetzen, welche der rechtlich erlaubten Beziehungen im Zusammenhang mit Drittmittel und Sponsoring zwischen Ärzteschaft und Industrie sie aus medizinischer und ethischer Sicht als wünschbar ansieht und welche nicht.

\section{Begriffsklärungen}

Unter Drittmitteln werden geldwerte Vorteile verstanden, welche einer Person oder einer Institution von externer Stelle unter Vereinbarung einer direk-

Revision des Strafgesetzbuches (StGB), in Kraft seit 1. Mai 2000. 
ten Gegenleistung (zweckgebunden, Projektfinanzierung) zur Verfügung gestellt werden und die für beide Parteien denselben Wert besitzen (im Gegensatz zu Sponsoring).

Unter Sponsoring ist die Vergabe geldwerter Vorteile ohne direkte gleichwertige Gegenleistung, mit oder ohne Auflagen zur Deklaration, zu verstehen.

Als Zuwendungen werden nachfolgend sowohl Drittmittel wie Sponsoring zusammengefasst.

Wichtig für das Verständnis der rechtlichen Fragen ist die Unterscheidung von zwei grundsätzlich verschiedenen Themenbereichen:

- Revidiertes Korruptionsstrafrecht. Der vorliegende Expertenbrief behandelt ausschliesslich diese Thematik. Dabei geht es um die Frage, wer welche Zuwendungen entgegennehmen darf. Das Gesetz betrifft nur die öffentlichen Spitäler (generell den Service public). Die Grundregel vorweg: Drittmittel und Sponsoring sind mit der seit 1. Mai 2000 geltenden Revision des Strafgesetzbuches nicht grundsätzlich problematisiert worden. Solche Zuwendungen dürfen entgegengenommen werden, aber sie müssen dem öffentlichen Spital bzw. seinen Kliniken oder Abteilungen zukommen, und nicht einer einzelnen Person (vgl. dazu nachfolgende Ziffern 3 bis 5).

- Definition und Weitergabe von Vergünstigungen an den Patienten. Empfehlungen zu dieser Frage sind im Expertenbrief nicht möglich. Es geht dabei um die korrekte Anwendung des Auftragsrechts und des Sozialversicherungsrechts, und nicht um das revidierte Korruptionsstrafrecht: Wann liegt ein Rabatt etc. vor, der an den Patienten oder den Versicherer weiterzugeben ist? Die Frage betrifft nicht nur die öffentlichen Spitäler, sondern alle Leistungserbringer, also auch Privatspitäler, Arztpraxen und alle andern Institutionen, die Patienten behandeln. Entscheidend ist bei dieser Thematik, dass sowohl vom konkreten ökonomischen

${ }^{2}[\ldots]$ und auch in den Beziehungen zu ausländischen Staaten: Schweizer Unternehmen sollen ausländische Regierungen nicht bestechen, um Aufträge hereinzuholen.

${ }^{3}$ Art. 15 Abs. 3 Universitätsförderungsgesetz des Bundes.

${ }^{4}$ Botschaft BBl 1999 S. 5536. Vgl. für zusätzliche Informationen den nachfolgenden Beitrag von Kuhn HP: Weiterführende rechtliche Informationen zu Drittmitteln, Sponsoring und Vergünstigungen.

${ }^{5}$ Neunzehnter Titel: Bestechung // Art. 322 series Vorteilsannahme:

"Wer als ... Beamter ...»: Gemeint ist der sogenannte "funktionale Beamte» - also Beamte, Angestellte etc. - aber immer nur diejenigen in einer Institution mit öffentlichem Auftrag.

${ }^{6}$ Neuer Art. 322 ${ }^{\text {octies }}$ Abs. 3: "Amtsträgern gleichgestellt sind Private, die öffentliche Aufgaben erfüllen.»

${ }^{7}$ Neuer Art. $322^{\text {series }}$ Vorteilsannahme:

"Wer als [...] Beamter [...] im Zusammenhang mit seiner amtlichen Tätigkeit für eine pflichtwidrige Tätigkeit oder eine im Ermessen stehende Handlung oder Unterlassung für sich oder einen Dritten einen nicht gebührenden Vorteil fordert, sich versprechen lässt oder annimmt, wird mit Zuchthaus bis zu fünf Jahren oder mit Gefängnis bestraft.»

${ }^{8}$ Definitionen des Bundesrats in der Botschaft, Bundesblatt (BBl) 1999, S. 5537 und S. 5550.
Kontext abhängt, ob eine Vergünstigung im Sinn von Auftragsrecht und KVG vorliegt (z.B.: Besteht ein massgeblicher Referenzpreis? Geht es um Einzelleistungstarife oder um Pauschaltarife oder um Lehre und Forschung?), wie auch, in welchem Umfang eine vorliegende Vergünstigung weiterzugeben ist (z. B.: Sind Entschädigungen für Kosten von Handling, Patienteninstruktion, Übernahme von Inkasso und Delkredererisiko, Verzinsung investierten Kapitals etc. zu berücksichtigen?). Diese Fragen können nur im Zusammenhang mit Tarifverhandlungen zwischen den Partnern im Gesundheitswesen geklärt werden.

\section{Die Bedeutung des revidierten Korruptions- strafrechts}

Die neue Fassung des schweizerischen Strafgesetzbuches ist seit 1. Mai 2000 in Kraft. Ziel der Revision war unter anderem eine wirksamere Bekämpfung der Korruption (Bestechlichkeit) in der Schweiz. ${ }^{2}$

Dabei ist dem Gesetzgeber die herausragende Bedeutung von Drittmitteln für die Forschung bewusst. So akzeptiert beispielsweise das neue schweizerische Universitätsförderungsgesetz Drittmittel nicht nur, sondern es macht sie unter anderem zum Kriterium für die Zuteilung von Bundessubventionen.

Das neue Strafrecht will denn auch drittmittelfinanzierte Forschung ${ }^{4}$ und Sponsoring ausdrücklich nicht verhindern. Entscheidend ist aber, dass alle Formen von Zuwendungen dem Spital zugute kommen, und nicht einer Einzelperson.

\section{Zentrale Elemente des neuen Strafrechts}

\section{Geltungsbereich}

Das neue Strafrecht betrifft nur das Personal ${ }^{5}$ der öffentlichen Institutionen (des Service public, also in der Medizin vor allem der öffentlichen Spitäler und der medizinischen Fakultäten), nicht aber Privatspitäler und Privatpraxen. Ebenfalls betroffen ist das Personal der Privatspitäler dann, wenn diese eine "öffentliche Aufgaben erfüllen». ${ }^{6}$ Die Rechtsform des Spitals spielt dabei keine Rolle.

\section{Inhaltliche Grundregel 1}

Strafbar sind geldwerte Zuwendungen (Drittmittel, Kongressbeiträge, Geschenke etc.), die nicht die öffentliche Institution, sondern eine Person für sich selbst erhält. ${ }^{7}$

\section{Inhaltliche Grundregel 2}

Nicht strafbar sind Geschenke an eine Einzelperson in der öffentlichen Institution, die "geringfügig und sozial toleriert" sind, die also "schon auf Grund gesellschaftlicher Normen keine Befangenheit hervorrufen können.» ${ }^{8}$ 


\section{Empfehlungen}

- Prüfen, ob Spital öffentliche Aufgabe erfüllt. Nötigenfalls unter Beratung durch die kantonale Gesundheitsdirektion feststellen, ob das Spital eine öffentliche Aufgabe im Sinne des neuen Strafrechts erfüllt. Faustregel: Entscheidendes Kriterium dürfte in der Regel sein, ob das Spital Subventionen erhält.

- Wenn öffentliche Aufgabe gegeben: alle Spitalabläufe durchleuchten im Hinblick auf die Frage, wer etwas persönlich erhält. Damit soll maximale Transparenz als Mittel zum Zwecke der Korruptionsprävention geschaffen werden.

\section{Empfehlungen für Massnahmen}

Die Kommission empfiehlt Spitälern und Ärzten vorerst die Umsetzung der nachstehend genannten Massnahmen $^{9}$ :

a) Positivliste kleiner Geschenke. Spitalintern bzw. fakultätsintern (allenfalls in Absprache mit der Gesundheits- bzw. Erziehungsdirektion) festlegen, welche Geschenke wann an wen als "geringfügig und sozial toleriert" gelten.

b) Festlegen: Grosse Zuwendungen nur melden oder genehmigen? Spitalintern bzw. fakultätsintern (allenfalls in Absprache mit der Gesundheits- bzw. Erziehungsdirektion) festlegen, ob der Erhalt von grösseren Zuwendungen nur zu melden oder ausdrücklich zu bewilligen ist: Wie und an wen melden? Gegebenenfalls: Genehmigung durch wen?

c) Festlegen: Auch geringfügige Geschenke melden? Spitalintern bzw. fakultätsintern entscheiden, ob auch die Zuwendungen gemäss Positivliste (a) gemeldet werden sollen; wenn ja: wie und an wen?

\footnotetext{
${ }^{9}$ Empfehlungen in Anlehnung an Prof. Dr. iur. Kurt Ulsenheimer: Industriesponsoring und Bestechlichkeit. Gastreferat am Deutschen Kongress für Gynäkologie und Geburtshilfe, München, 15.6.2000, und basierend auf einer Motion der Kommission für Rechtsfragen des Nationalrats, Oktobersession 1999.
}

d) Strikte Trennung von Firmenzuwendung und Bestellung, d.h. Entkoppelung von Vorteil und Firmenauftrag.

e) Kontendistanz, d.h., nicht der Arzt, sondern ein Dritter soll die Verfügungsmacht über das Konto besitzen.

f) Kollektivunterschrift und Vier-Augen-Prinzip: Bei allen grösseren Einkäufen und Aufträgen Kollektivunterschrift im Spital bzw. in der Fakultät einführen; Kontrollen immer durch zwei Personen (= wer nicht allein entscheiden kann, ist weniger anfällig).

g) Schriftlichkeit aller Absprachen über Zuwendungen.

h) Keine Bargeldannahmen, d.h. Zuwendungen nur in Form von Überweisungen oder Verrechnungsschecks.

i) Bei der Publikation von Forschungsarbeiten Drittmittel deklarieren.

\section{Schlussbemerkungen}

Alle bekannten Kodizes zum ärztlichen Berufsethos verlangen vom Arzt eine Unterordnung persönlicher Interessen unter diejenigen seiner Patienten. Tritt ein Dritter, wie z. B. ein Sponsor, in Kontakt mit dem Arzt, so kann beim Patienten und in der Öffentlichkeit die Angst aufkommen, dass Entscheidungen des Arztes bzw. die ursprüngliche Arzt-Patienten-Beziehung beeinflusst werden könnten. Gerade im Bereiche möglicher finanzieller Interessenskonflikte vermag deshalb nur maximale Transparenz der Entstehung von Misstrauen vorzubeugen.

Die Diskussion über die Beziehung zwischen Ärzteschaft und Industrie ist angesichts ihrer Aktualität dringend und umfassend zu führen. Die Revision des Korruptionsstrafrechts ist dabei nicht als Bedrohung, sondern als Chance zu sehen: Sie verlangt und fördert Transparenz, die dem Vertrauen in die Medizin und in die notwendigen Beziehungen zwischen Industrie und Medizin dient. 